\title{
A CIÊNCIA INVISÍVEL: POR QUE OS PESQUISADORES NÃO PUBLICAM SEUS RESULTADOS NEGATIVOS?
}

\section{THE INVISIBLE SCIENCE: WHY RESEARCHERS DO NOT PUBLISH THEIR NEGATIVE RESULTS?}

\author{
Luís Fernando Sayãoa \\ Luana Farias Sales ${ }^{b}$
}

\begin{abstract}
RESUMO
Introdução: Os resultados de pesquisas cientificas considerados negativos - hipóteses não confirmadas, dados inesperados, experimentos não concluídos e outros mais estão sempre presente nos fluxos de construção do conhecimento científico, constituindo um componente essencial para uma visão mais abrangente e integral das múltiplas faces da rotina dos laboratórios. Além do mais, são esses estudos que refutam as ideias correntes e hipóteses consolidadas que fazem a ciência avançar. Entretanto, os estudos negativos não encontram meio de publicação formal de alto impacto e não são plenamente valorizados no ambiente científico e pelas suas instituições. São muitas as barreiras para a não publicação e compartilhamento desses resultados. Objetivo: Tentando compreender esse fenômeno, o presente estudo tem por objetivo identificar as principais causas da invisibilidade dos estudos negativos. Metodologia: A partir de uma pesquisa exploratória e qualitativa, adota-se como recurso metodológico, a busca e a análise da escassa literatura sobre a temática, investigando e sistematizando as principais causas da invisibilidade dos estudos negativos. Resultado: Como resultado, apresenta-se essas causas categorizadas e comentadas em cinco categorias: barreiras contextuais, cognitivas, profissionais, organizacionais e editoriais. Conclusões: Conclui-se que embora haja um movimento em torno da criação de periódicos e de outras plataformas dedicadas aos resultados negativos, esses ativos informacionais ainda precisam ser valorizados e integrado aos fluxos de comunicação científica proporcionando maior visibilidade aos estudos não confirmatórios.
\end{abstract}

Descritores: Resultado negativo. Periódicos científicos. Comunicação científica. Ciência invisível.

a Doutor em Ciência da Informação pelo Instituto Brasileiro de Informação em Ciência e Tecnologia / Universidade Federal do Rio de Janeiro (PPGCI-IBICT-UFRJ). E-mail: Isayao@cnen.gov.br

b Doutora em Ciência da Informação pelo Instituto Brasileiro de Informação em Ciência e Tecnologia / Universidade Federal do Rio de Janeiro (PPGCI-IBICT-UFRJ). Coordenadora Geral de Acesso e Difusão de Acervos do Arquivo Nacional. Docente do Programa de Pósgraduação em Ciência da Informação do convênio IBICT-UFRJ. E-mail: luanafsales@gmail.com 


\section{INTRODUÇÃO}

E O Editorial Rewarding negative results keeps science on track (2017), de 23 de novembro, da revista Nature, repercute dois prêmios científicos que sinalizam uma mudança na escala de avaliação dos resultados de pesquisa denominados de "não confirmatórios". Um deles, patrocinado pelo European College of Neuropsychopharmcology', ofereceu um prêmio de dez mil euros para trabalhos que relatassem resultados negativos em neurociência pré-clínica: o prêmio era destinado a experimentos cuidadosos que não confirmam uma hipótese aceita ou um resultado prévio. O outro, oferecido pela Organization for Human Brain Mapping², seguiu os mesmos critérios e já está na segunda edição. Os ganhadores são selecionados pela qualidade dos estudos e pela importância dos achados que estavam sendo avaliados, ainda que enquadrados como "resultados negativos" pela comunidade científica.

Os anúncios dos prêmios, em conjunto com outras ações ainda mais relevantes, colocam em pauta as discussões sobre o reconhecimento, por parte de setores específicos da comunidade científica - como as agências de fomento à pesquisa e de algumas casas editoriais -, da importância dos resultados negativos para o avanço da ciência e para a construção de uma visão mais abrangente e completa dos múltiplosângulos da pesquisa científica. Os resultados de experimentos científicos considerados negativos - hipóteses não confirmadas, dados inesperados, experimentos não concluídos e outros mais constituem uma parte inseparável do cotidiano dos laboratórios e um componente essencial da integralidade dos fluxos de geração de conhecimento.

Há um consenso claro - atestado pela literatura - de que grande parte das pesquisas produz achados não confirmatórios ou negativos. São pesquisas que desafiam modelos, princípios e dogmas correntes baseados em estudos rigorosos e bem documentados (PFEFFER; OLSEN, 2002). Essas observações refutam ideias correntes e hipóteses cuidadosamente construídas e

\footnotetext{
1 Disponível em: https://acnp.org/. Acesso em: 10 dez. 2019.

2 Disponível em:

https://www.humanbrainmapping.org/i4a/pages/index.cfm?pageid=3267\&pageid=1. Acesso em: 10 dez. 2019.
} 
consolidadas que fazem a ciência avançar. "Pode-se argumentar que 'dados negativos', longe de terem pouco valor para a ciência, são atualmente uma parte integral do progresso científico que merecem mais atenção", destacam Pfeffer e Olsen (2002). Anderson, Olsen e Sprott (2013, p. 1) sinalizam a necessidade de incorporação desses resultados aos esquemas acadêmicos de publicação e recompensa, redefinindo uma ecologia de achados científicos mais diversificada, abrangente e menos distorcida pelo "viés positivo".

Não obstante o reconhecimento por parte de algumas comunidades científicas da importância dos resultados negativos, para um retrato mais fiel das múltiplas atividades de pesquisa, esses produtos de pesquisa não encontram meios de publicação formal de alto impacto e não são ainda plenamente valorizados no meio acadêmico e nem pelas agências de fomento. A invisibilidade sobre os resultados negativos tem efeito crucial sobre os princípios de autocorreção da ciência, da reprodutibilidade dos experimentos científicos e da transparência de seus processos. Isso sem falar na duplicação de esforços financeiros e intelectuais desperdiçados na repetição de experimentos que são becos sem saída; nos desvios éticos causados pelo chamado "viés de publicação" (publicationbias) ${ }^{3}$, que cria uma inclinação artificial para resultados positivos que são mais facilmente publicáveis; e ainda tornam mais evidentes questões contemporâneas críticas como, por exemplo, o sacrifício desnecessário de animais.

São muitos os motivos para a não publicação e compartilhamento dos resultados negativos. É um problema de muitas faces cuja compreensão se inicia com a própria idealização do trabalho científico; passa pela vaidade, crença e incertezas profissionais e acadêmicas dos pesquisadores; e vão até a concorrência dos editores por resultados espetaculares e pela compreensão equivocada dos agentes financiadores de pesquisa e dos centros de pesquisa sobre a importância dos resultados negativos. Essa associação de motivos e percepções dos diversos atores envolvidos contribui para que uma parcela importante dos achados científicos jamais veja a luz do sol e permaneça, para

\footnotetext{
${ }^{3}$ Disponível em: https://pt.wikipedia.org/wiki/Vi\%C3\%A9s_de_publica\%C3\%A7\%C3\%A3o. Acesso em: 10 dez. 2019.
} 
sempre, como um iceberg de conhecimento, submerso nas gavetas dos laboratórios e nos computares pessoais dos pesquisadores.

Compreendendo a criticidade desse fenômeno para a Ciência da Informação, o presente ensaio procura analisá-lo segundo a perspectiva da comunicação científica, identificando e sistematizando os diversos obstáculos que impedem a publicação e o compartilhamento de resultados de pesquisa considerados negativos. Para tal, tomou-se como metodologia a análise da escassa literatura formal sobre o assunto, além de blogs, fóruns de discussão, ensaios, editoriais e comentários.

\section{A IMPORTÂNCIA DOS RESULTADOS NEGATIVOS PARA A CIÊNCIA}

A ciência contemporânea, moldada mais e mais pelas tecnologias digitais e pela velocidade e instantaneabilidade das redes, torna-se essencialmente um empreendimento colaborativo, virtualizado, onipresente e distribuído globalmente. Isto acontece por vários motivos: seja pelos pressupostos da ciência aberta que se impõe com seus signos humanísticos de compartilhamento do conhecimento científico como um bem comum; seja pelo uso intensivo de processos de simulação por computação dos fenômenos da natureza e da sociedade - 0 deslocamento da ciência do in vitro para in silico; ou ainda pela impossibilidade de compreensão e análise, por um único laboratório, por exemplo, dos petabytes ${ }^{4}$ de dados gerados pelos aparatos científicos modernos (SAYÃO; SALES, 2019). Nesse contexto, cujo excesso de dados e informações é mais crítico do que a sua escassez, os fluxos de compartilhamento e de interlocução tornam-se essenciais na convergência para o conhecimento consensual, ainda mais considerando que os projetos científicos raramente resultam em descobertas perfeitas sobre elementos do universo, da natureza e da sociedade. Seus argumentos precisam estar expostos ao confronto e ao debate.

Assim sendo, o debate dos argumentos se torna parte dos percursos científicos, como enfatiza Bruno Latour (2017, não paginado): "O objetivo da

\footnotetext{
${ }^{4}$ Um petabyte corresponde a $10^{15}$ bytes de informação digital. Disponível em: https://en.wikipedia.org/wiki/Petabyte. Acesso em: 10 dez. 2019.
} 
ciência não é produzir verdades indiscutíveis, mas discutíveis. Nem as ciências naturais e exatas produzem verdades indiscutíveis. [...] O objetivo é produzir discussões públicas normatizadas", completa o autor.

Essa trajetória dialética de erros e acertos está evidente no ciclo contínuo de confrontos e rupturas que conta a história da ciência. São os achados negativos que levam à refutação de hipóteses; que obrigam os cientistas a uma reflexão crítica sobre a validade do pensamento corrente; que revelam novas visões e que, por fim, revolucionam a ciência. $O$ confronto e a refutação é o moto contínuo da geração do conhecimento científico.

O filósofo Karl Popper (1962) e o também filósofo e físico Thomas Kuhn (1970) exaltam a importância da refutação para o progresso da ciência. A transição entre os paradigmas do pensamento corrente - por mais duradoura que ela seja - e as novas concepções fascinavam esses autores, que registraram suas ideias nos seus livros, que são clássicos imprescindíveis para os estudos da filosofia da ciência. São eles, respectivamente, Conjecture and refutation: The growth of scientific knowledge, publicado em 1963 e The structure of scientific revolution, de 1970.

Popper, com o intuito de mostrar que a ciência avança por meio de processos de "conjecturas e refutação", ofereceu um exemplo simples e fascinante, que foi relatado por Pfeiffer e Olsen (2002, p. 1): durante centenas de anos - baseados na observação de milhões de cisnes - os europeus acreditavam que os cisnes eram sempre brancos. Isso aconteceu até que a exploração da Australásia apresentou aos europeus os cisnes negros. Bastou um único cisne negro para repudiar a teoria deque todos os cisnes eram brancos. "Apesar de muitos exemplos confirmatórios existirem para manter uma teoria, basta uma contra observação para refutá-la" (PFEIFFER; OLSEN, 2002, p. 1).

Por mais fascinantes que sejam os argumentos de Popper, a realidade mostra que, historicamente, os cientistas que chegam a resultados que se contrapõem aos paradigmas estabelecidos precisam travar muitas batalhas para alcançar o reconhecimento por suas descobertas. Alguns exemplos que ficam como uma consagração à refutação são os icônicos experimentos não confirmatórios realizados no século XIX por Albert Michelson e Edward Morley para provar a 
existência do éter luminífero- um meio teórico através do qual se propagariam as ondas eletromagnéticas. Mais tarde, esses experimentos inspiraram Einstein a desenvolver a teoria da relatividade (EINSTEIN; INFIELD, 1936) e contribuíram para que Michelson fosse laureado com o Prêmio Nobel de Física de 1907.

Porém, a grande maioria desses estudos permanece na obscuridade, quando não é ridicularizada, outem suas descobertas inovadoras confirmadas somente décadas depois. As pesquisas dobotânico Gregor Mendel ${ }^{5}$ considerado pai da Genética -, ilustram bem esse fato. Durante sete anos o cientista extraiu meticulosamente dados a partir de centenas de cruzamentos, em um universo de 29 mil pés de ervilhas cultivados por ele. $O$ objetivo era investigar como as características das plantas eram passadas de geração para geração; partindo daí deduziu o que ele chamou de Primeira e Segunda Lei da Hereditariedade. Mendel ainda formulou um modelo simples pelo qual essas leis poderiam operar e propôs que os traços observados são determinados por "fatores" discretos que hoje são conhecidos como genes. No período entre 18651867 os resultados de suas pesquisas foram apresentados às sociedades científicas e autoridades, mas foram, todavia, ignorados por desafiarem as teorias contemporâneas. Não antes de 1902, quando Hugo de Vries, Carl Carrens e Erich von Tschermark redescobriram os princípios formulados por Mendel, que o ramo da Biologia conhecido como Genética teve início (PFEIFFER; OLSEN, 2002; MOSLEY; LINCH, 2011).

Nesse confronto dialético, um aspecto essencial da ciência é a sua suposta transparência em apresentar todas as faces da história de um achado científico (MATOSIN et al., 2014), isto, idealmente, inclui as experiências, percursos, tentativas que coletivamente contribuíram para a construção consensual do conhecimento científico. A pesquisa científica perde seu poder de autocorreção e fragiliza o princípio de reprodutibilidade quando há informações faltando sobre esse percurso (BAKER, 2016).

Os achados não confirmatórios resultam na rejeição de hipóteses consolidadas. Porém, apesar do reconhecimento de que são eles que

\footnotetext{
${ }^{5}$ Disponível em: https://pt.wikipedia.org/wiki/Gregor_Mendel. Acesso em: 6 dez. 2019.
} 
impulsionam o progresso da ciência, grande parte desses percursos investigativos não são documentados. No cotidiano dos laboratórios, os resultados negativos permeiam todo o ciclo da pesquisa científica, e constituem uma parte importante da integralidade dos fluxos da investigação científica. "Resultados nulos, negativos ou inesperados são ocorrências possíveis para pesquisadores em todo o mundo", confirmam Guimarães et al. (2018, p. 1). "Atualmente, as descobertas científicas publicadas representam somente 10\% dos estudos realizados, $90 \%$ são de resultados negativos não publicados. A perda de informação é imensa", estima com maior contundência o editorial da Recherche Animale (2018, p. 1). Mesmo diante do pressuposto de que o método científico é absoluto e que os experimentos foram bem projetados e controlados, seus resultados, porém, podem não ser relatados jamais.

Como consequência, os resultados de milhares de experimentos negativos, muitos deles de grande importância para o progresso da ciência, jamais serão conhecidos e permanecerão para sempre fechados nos cadernos de laboratórios, gavetas e computadores pessoais dos pesquisadores, onde serão, inevitavelmente, tragados pelo tempo, pela obsolescência tecnológica e pela fragilidade intrínseca das mídias digitais.

A perspectiva triunfalista da ciência, que se volta de forma privilegiada para os produtos finais da pesquisa - o invento, o artigo, a patente, a tese - condena à obscuridade tudo o que não é sucesso e que está inconcluso, e deixa sem registro uma parte relevante dos labirintos trilhados pelos cientistas até o produto final da pesquisa. Este contexto científico induzido pelo viés de resultados positivos tem um rebatimento claro nos fluxos de comunicação científica, mais especificamente nas publicações acadêmicas, que somente em alguns casos aceitam resultados negativos, sob o argumento do baixo impacto desses relatos.

Nesse contexto moldado pelo viés positivo, o desafio de se opor às hipóteses estabelecidas e prevalentes por meio de novas pesquisas impõe a necessidade de se romper muitas barreiras - cognitivas, comportamentais, organizacionais e sociais. Isto acontece porque os resultados não confirmatórios são frequentemente associados a estudos metodologicamente falhos ou mal projetados que têm um impacto negativo sobre a carreira do pesquisador e são, 
via de regra, mal considerados por editores e agências de fomento à pesquisa.

São essas as questões que discutiremos a seguir.

\section{POR QUE OS PESQUISADORES NÃO PUBLICAM SEUS RESULTADOS NEGATIVOS?}

O fenômeno do baixo interesse pelas publicações de resultados negativos tem muitas faces, entretanto, nenhuma delas o explica completamente. A pluralidade de motivos para a invisibilidade desses resultados reflete complexidade sociológica, meritocrática, econômica e comportamental das relações do universo da ciência e de seus diversos atores, que são, finalmente, pessoas imersas em ambientes de profundas interações humanas. Partindo da análise dos autores que trataram do assunto, foram identificadas e sistematizadas na figura 1 as principais barreiras que levam os pesquisadores a não publicarem os resultados negativos de suas pesquisas.

Figura 1 - Barreiras na publicação de resultados negativos

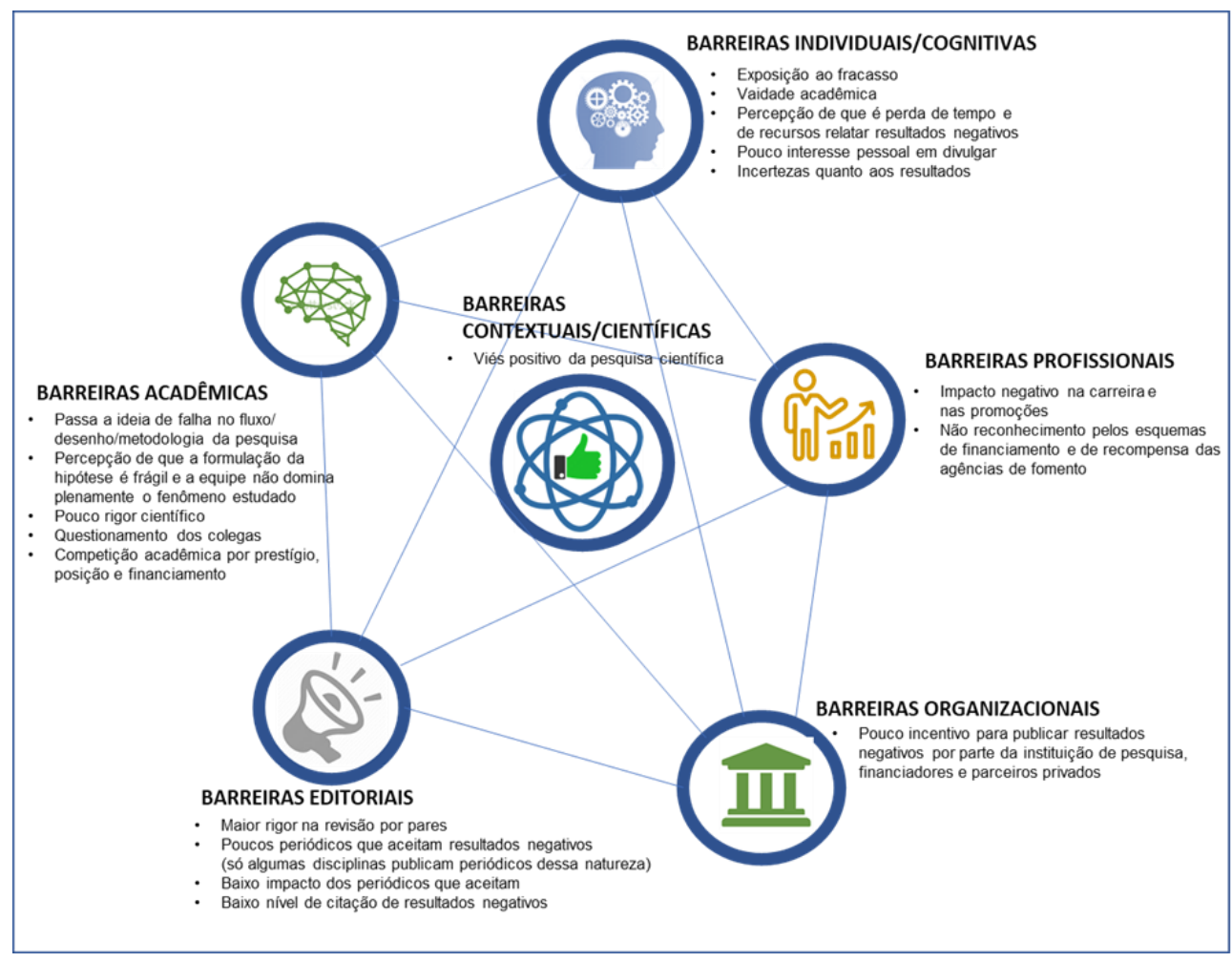

Fonte: elaborada pelos autores 


\subsection{BARREIRAS CONTEXTUAIS}

Compreendem as barreiras impostas pela própria ciência e sua idealização como atividade humana nobre, que valoriza prioritariamente os empreendimentos e as descobertas científicas conclusivas e acabadas. Considera também a visão frequentemente romantizada da ciência como um sistema de construção de conhecimento infalível, onde cientistas trabalham em colaboração para sistematicamente acharem respostas precisas sobre 0 universo (MATOSIN et al., 2014).

Esta perspectiva simbolicamente triunfalista da ciência cria, no final de tudo, uma inclinação para a publicação de resultados positivos, e negligencia um aspecto fundador da ciência que é o de apresentar todas as faces da história de um achado científico (MATOSIN et al., 2014) e de colocar seus princípios sempre em reconsideração acionados pela "discutibilidade" dos argumentos da ciência (LATOUR, 2017).

O fato de que há ocasiões em que novas evidências refutam antigas hipóteses cuidadosamente estabelecidas e impactam o pensamento corrente, por mais duradouro que ele seja, fazem a ciência revolucionar a si mesma. Essa ruptura é um desafio importante que deve ser superado e celebrado, em nome do progresso da ciência, como um elogio à refutação. Porém, como observado no presente estudo, a ótica conservadora da ciência impacta de forma contundente a publicação dos resultados negativos.

Popper (1962) e Kuhn (1970) elegem as rupturas paradigmáticas como pontos de inflexões essenciais para o progresso da ciência. Eles deixam explicito, entretanto, que o desafio de se contrapor a visões científicas consolidadas por meio de resultados disruptivos, impõe a necessidade imensa de se romper as barreiras cognitivas, comportamentais, econômicas e sociais intrínsecas à organicidade da teia da ciência. "Em face ao desafio de se contrapor à visão corrente por meio de novas pesquisas, nosso próprio viés cognitivo torna difícil reunir forças para lutar pela mudança de paradigma", resumem MATOSIN e seus colaboradores (2014, p. 1) o dilema dos pesquisadores.

É nessa direção que a discussão prossegue. 


\subsection{BARREIRAS INDIVIDUAIS E COGNITIVAS}

Estão relacionadas ao quadro de crenças e atitudes individuais dos pesquisadores em relação aos seus resultados negativos e ao ambiente científico em que estão inseridos.

Mesmo diante do fato comprovado de que os resultados nulos, negativos ou inesperados são ocorrências possíveis que fazem parte do cotidiano dos laboratórios e, na maioria dos casos, ocorrem com maior frequência do que os resultados positivos, muitos pesquisadores desenvolvem a atitude de que publicar resultados negativos é um desperdício de recursos e de tempo (HENDRIX, [2016]).

Como colocado por Fanelli (2010), na condição de seres humanos, os cientistas são tendenciosos para a confirmação - isto é, selecionam informações que ratificam a sua visão sobre o seu universo de pesquisa, criando um viés positivo para suas investigações. "Portanto, os resultados negativos são uma verdade inconveniente, e ignorar os resultados inconsistentes [com os paradigmas correntes] seria apenas parte da condição humana", resumem Matosin et al. (2014, p. 171).

É ainda necessário enfatizar que num ambiente inerentemente competitivo é de se esperar que os pesquisadores relutem em expor seus supostos fracassos, que possam gerar questionamentos e incertezas por parte de seus pares. Este padrão de comportamento é reforçado pelas respostas dos demais atores e das instituições que se inter-relacionam no mundo da ciência, e que se apresentam na forma de reconhecimento, financiamento, posição, citação, revisão por pares e publicação de impacto. Entra em jogo também a vaidade, ingrediente relevante no mundo da pesquisa, que faz com que muitos pesquisadores escolham não prosseguir com achados não confirmatórios, porque comprovadamente eles geram pouco interesse científico e poucas citações que, consequentemente, têm desdobramentos na vida profissional.

Por fim, van Hilten (2015) nos alerta que a linguagem em torno dos achados negativos cria um efeito depreciativo, citando a declaração de um pesquisador: "Chamando esses resultados de 'negativos' eles se tornam imediatamente menos atrativos - especialmente para os pesquisadores, que 
geralmente preferem não publicá-los" (VAN HILTEN, 2015, p. 6).

\subsection{Barreiras ProfissionaIS}

Estão relacionadas à percepção, por parte dos pesquisadores, sobre os impactos - reais e imaginários - que a publicação de resultados negativos tem sobre a sua carreira profissional em termos de promoção, de recompensa e de financiamento de suas pesquisas.

A análise realizada por Jian Tang e Ranata Curty, citados por Gabriella Anderson (2012), destaca alguns fatores bastante contundentes. Uma das suposições alinhadas pelos autores é a deque a publicação de resultados negativos pode arruinar a reputação dos pesquisadores, posto que podem, indiretamente, transmitir para a comunidade científica a ideia de que o trabalho foi mal planejado e que os pesquisadores não conhecem o bastante sobre o fenômeno estudado ou são incapazes de formular uma hipótese robusta que oriente a pesquisa.

Os motivos profissionais são também discutidos longamente por Hendrix (2016), que afirma que muitos orientadores estão convencidos de que a publicação de estudos negativos pode fragilizar a trajetória acadêmica de seus estudantes de doutorado e, ainda, suas próprias carreiras. "Eles despenderão muitos recursos num projeto errado, publicando com baixo impacto e consequentemente obtendo menos aportes financeiros no futuro" (HENDRIX, 2016).

Muitas carreiras, como postos na indústria ou no setor público, as publicações de alto fator de impacto não são absolutamente necessárias, porém "fatores de impacto ainda são amplamente usados para avaliar o desempenho de cientistas, departamentos e instituições [de pesquisa]" (HENDRIX, 2016).

\subsection{BARREIRAS ORGANIZACIONAIS}

Dizem respeito ao posicionamento das instituições de pesquisa e de fomento e de outras instituições que dialogam como mundo da ciência (empresas, sociedades científicas, ONG's etc.) no dimensionamento das 
pesquisas que geram resultados negativos. O reconhecimento dessas instituições, em termos de recompensa dos resultados de pesquisa, se apresenta na forma de financiamento, posição e prestígio. Suas métricas são baseadas fortemente em publicação de impacto e no número de citações.

Dessa forma, muitos pesquisadores escolhem não prosseguir com achados não confirmatórios, não só porque eles geram pouco interesse científico e poucas citações, mas também pela resposta pífia das instituições ao esforço de pesquisa que se efetivam em resultados negativos. Como consequência, a quantidade de relatos sobre dados não significativos vem progressivamente declinando (MATOSIN et al., 2014; FANELLI, 2012).

A competição é encorajada no universo científico especialmente em países cientificamente avançados, pois considera-se que ela aumenta a eficiência e a produtividade dos pesquisadores (FANELLI, 2010), entretanto "quando a concorrência é pervasiva, seus efeitos podem comprometer o progresso, a eficiência e a integridade da ciência", destacam Anderson, Olsen e Sprott (2007, p. 1), corroborando os efeitos adversos de uma ciência seletiva e parcial.

\subsection{BARREIRAS ACADÊMICAS}

Dizem respeito ao julgamento dos processos de pesquisa que geram resultados negativos, tais como, workflow, desenho da pesquisa, formulação de hipóteses, metodologia e, seus efeitos na avaliação por pares. Tang e Curty, citados por Gabriella Anderson (2012, p. 1), destacam que há uma maior probabilidade de que originais que reportam resultados negativos sejam recusados na revisão por pares, dada a percepção de falta de solidez em comparação com estudos que apresentam resultados "bem-sucedidos". Este argumento é reforçado por Hendrix (2016), que nos alerta que os revisores acadêmicos tendem a impor controles adicionais aos resultados negativos para terem certeza de que não se trata somente de efeitos de erros técnicos ou metodológicos.

Esta condição pode induzir o pesquisador a "distorcer o conhecimento, particularmente quando os resultados obtidos não são aqueles esperados", 
como assinalam Guimarães e seus colaboradores (2018, p. 246), produzindo, dessa forma, resultados espúrios, enviesados em direção a resultados que sejam melhores aceitos pelos códigos editoriais. Confrontado com um resultado negativo, portanto, um cientista pode ser tentado a não perder tempo em publicálo - comportamento conhecido como "efeito arquivo de gaveta"; ou torná-lo positivo, por meio da reformulação das hipóteses iniciais, selecionando os resultados que devem ser publicados, reformulado os dados ou as análises, entre outras estratégias. Estes procedimentos são conhecidos internacionalmente pela sigla em inglês HARKing - Hypothesizing After the Results are Known (FANELLI, 2010).

\subsection{BARREIRAS EDITORIAIS}

Estão relacionadas à precariedade da infraestrutura editorial voltada para a publicação de resultados negativos que se consubstanciam na escassez de periódicos e de outras plataformas que aceitam publicar esses resultados. Considera também o relativo baixo impacto dos periódicos que publicam resultados negativos.

As observações levantadas por ocasião do lançamento, em 2002, do periódico Journal of Negative Results in Bimedicine ${ }^{6}$, destacadas no editorial de seu primeiro número por Pfeffer e Olsen (2002, p. 1), revelam com clareza o posicionamento crítico e as dúvidas sobre os periódicos que publicam resultados negativos, por parte dos editores e da comunidade científica:

[...] Por que esse tipo de periódico? Quais são os benefícios de um periódico que publica resultados negativos? Essas informações publicadas não darão vantagem aos meus concorrentes? Como você vai evitar a publicação de ciência de baixa qualidade?

Não há exagero ao se afirmar que são escassos os meios de publicação acadêmica que aceitam resultados negativos, apesar das dezenas de milhares de periódicos acadêmicos em circulação (MUDRAK, 2019). Isto inclui repositórios voltados para a publicação de outros produtos de pesquisa, especialmente dados de pesquisa.

\footnotetext{
${ }^{6}$ Disponível em: https://jnrbm.biomedcentral.com/. Acesso em: 6 abr. 2020.
} 
A insuficiente literatura sobre os fenômenos relacionados aos resultados de pesquisa negativos é unânime em confirmar o fato de que esses resultados têm três vezes menos chances de serem publicados em periódicos científicos quando comparado aos resultados positivos (DICKERSIN et al., 1987; FANELLI, 2010; FANELLI, 2012; MATOSIN et al., 2014; HENDRIX, [2016]; TIMBIE, 2019). Além do mais, os poucos artigos acadêmicos que reportam resultados que falham em sustentar as hipóteses que estão sendo testadas, quando porventura publicados, têm menos oportunidades de serem citados (FANELLI, 2010). No mesmo sentido, Mudrak (2019) confirma essa constatação, registrando que os periódicos que aceitam resultados negativos não são intensamente utilizados e apresentam baixos níveis de citação para esses estudos. Como desdobramento imediato, há um rebatimento dessa performance no comportamento cauteloso de outros periódicos ao requisitarem artigos similares.

Num ambiente cuja competição é institucionalizada e que se efetiva na forma de parâmetros, padrões e métricas voltadas para avaliar o nível de impacto e de presença midiática, é de se esperar que os periódicos prefiram publicar resultados de estudos que gerem conclusões e produtos acabados que garantam maior impacto. Incluem-se nesse ponto os desdobramentos inapropriados dos efeitos dramáticos esperados pelos editores, tendo em vista que "a tendência corrente focada em resultados espetaculares pode levar a falsas conclusões", como alerta Mudrak (2019, p. 4). Periódicos de alto impacto podem ocasionalmente se interessar por estudos negativos, isso acontece quando esses resultados refutam paradigmas há muito tempo consolidados, ou quando um novo método é usado para mostrar que os estudos prévios são falhos.

Não há uma relação lógica entre resultados positivos e resultados de alto impacto, entretanto parece que a cultura científica considera que eles são análogos (MATOSIN et al., 2014). Críticos do statusquo da ciência vêm a longo tempo lamentado a tendência dos periódicos de não publicar estudos negativos, posto que isto não significa que os cientistas falham em encontrar efeitos em tais estudos. Muito pelo contrário: uma pesquisa, por exemplo, que descobre que uma droga não combate uma infecção pode ser uma informação importante e 
acionável. "Isso enfatiza que precisamos de esforços e incentivos sérios para publicação de estudos negativos, no sentido de ajudar a equilibrar os apoios diretamente dirigidos às publicações positivas", ponderam Oransky e Marcus (2016, p. 1).

Contudo, o reconhecimento sobre a importância do problema perece estar crescendo no meio acadêmico, com variado grau de sucesso que depende fortemente de domínios disciplinares específicos. Em algumas áreas, por exemplo, há cientistas que já estão argumentando que a exigência de revelar os resultados negativos decorrentes de um experimento deve ser uma condição para a publicação de um achado positivo (KNIGHT, 2003).

\section{4 À GUISA DE CONCLUSÃO}

O surgimento de plataformas para a publicação de resultados negativos, como periódicos científicos e repositórios, prêmios que laureiam pesquisas não confirmatórias conduzidas com rigor científico, fóruns de discussões sobre a questão, editoriais e blogs parece indicar um reconhecimento, por parte de setores específicos da comunidade científica e de algumas casas editoriais, da importância dos resultados negativos para o avanço da ciência e para a construção de uma visão mais completa das múltiplas faces da geração de conhecimento científico. Há, felizmente, um movimento para negar a negatividade.

Esta nova perspectiva redimensiona e recontextualiza termos como "positivo", "significante", "negativo", "resultado nulo", tão comuns no jargão científico enquanto qualificadores dos produtos de pesquisa. O que se observa é que estes atributos têm sido obviamente mal interpretados, posto que todos os resultados são igualmente relevantes para a ciência, na medida em que eles tenham sido produzidos por métodos lógicos, sólidos e aceitos pela comunidade científica (FANELLI, 2010).

Uma nova visão sobre os estudos negativos vem lentamente desencadeando movimentos e ações que podem torná-los mais visíveis e contornar o pessimismo que envolve esses produtos de pesquisa (MATOSIN et al., 2014). Nesse sentido, alguns periódicos - especialmente em algumas áreas 
específicas, como Biomedicina - foram criados especialmente para publicar experimentos não confirmatórios; além disso, periódicos convencionais abrem espaços para publicá-los ou publicam números especiais para resultados negativos; também surgem repositórios e outras plataformas com o mesmo propósito.

Entretanto, apesar da disponibilidade - ainda insipiente - de títulos de periódicos que publicam resultados negativos, frequentemente eles não atraem muitos artigos, demonstrando que são as culturas subjacentes às comunidades científicas e os quadros referenciais e cognitivos que delineiam as atitudes e comportamentos dos pesquisadores em relação ao problema, e que requerem mudanças, e não somente o contexto das políticas editoriais, organizacionais e de financiamento à pesquisa.

Estudos negativos ainda constituem uma baixa prioridade para a publicação acadêmica, mas é também uma baixa prioridade para a Ciência da Informação. É necessário enfatizar que o problema levantado está localizado no coração da comunicação científica, porém é quase que totalmente esquecido pelos estudos na área de Ciência da Informação. São poucos os autores que analisaram o fenômeno sob a perspectiva da área.

\section{AGRADECIMENTO}

Ao Conselho Nacional de Desenvolvimento Científico e Tecnológico CNPq pelo apoio a presente pesquisa.

\section{REFERÊNCIAS}

ANDERSON, Gabriella. No result is worthless: the value of negative results in science. On Medicine Blog. New York, 10 Oct 2012. Disponível em http://blogs.biomedcentral.com/on-medicine/2012/10/10/no-result-is-worthlessthe-value-of-negative-results-in-science/. Acesso em: $10 \mathrm{dez} .2019$.

ANDERSON, Gabriella; OLSEN, Bjorn R.; SPROTT, Haiko. Opinion: Publish negative results. The Scientists, [S.I.], 2013. Disponível em: https://www.thescientist.com/opinion/opinion-publish-negative-results-39928. Acesso em: 6 abr. 2020. 
BAKER, Monya. Is there a reproducibility crisis? Nature, [S.I.], v. 533, p. $452-$ 454, 26 May 2016. Disponível em:

https://www.nature.com/news/polopoly_fs/1.19970!/menu/main/topColumns/top LeftColumn/pdf/533452a.pdf. Acesso em: 10 dez. 2019.

DICKERSIN, K.; CHAN, S.; CHALMERSX, T. C.; SACKS, H. S.; SMITH JR, H. Publication bias and clinical trials. Controlled Clinical Trials, [S.I.], v. 8, n. 4, p. 343 - 353, 1987. Disponível em:

https://www.sciencedirect.com/science/article/abs/pii/0197245687901553. Acesso em: 6 ago. 2019.

EINSTEIN, Albert; INFIELD, Leopold. Lévolution des idées en physique. Paris: Flammarion, 1936.

FANELLI, Daniele. Do Pressures to Publish Increase Scientists' Bias? An Empirical Support from US States Data. PLoS ONE, [S.I.], v. 5, n. 4, e10271, 2010. Disponível em:

https://journals.plos.org/plosone/article?id=10.1371/journal.pone.0010271. Acesso em: 6 ago. 2019.

FANELLI, Daniele. Negatiive results are disappearing from most disciplines and countries. Scientometrics, [S.I.], v. 90, n. 3, p. 891-904, 2012. Disponível em: https://link.springer.com/article/10.1007/s11192-011-0494-7. Acesso em: 6 ago. 2019.

GUIMARÃES, Renan Arthur Bosio; SUCCI, Guillerme de Menezes; MONTALLI, Victor Angelo Martins; NIEDERAUER, Ana Júlia Schmidt; SUCCI, Regina Célia de Menezes. Resultados negativos na pesquisa científica: aspectos éticos.

Revista Bioética, Brasília, v. 26, n. 2, 2018. Disponível em:

http://www.scielo.br/scielo.php?pid=S1983-

80422018000200245\&script=sci_arttext. Acesso em: 6 ago. 2019.

HENDRIX, Sven. Should I publish negative results or does this ruin my career in science? [2016]. Disponível em:

https://smartsciencecareer.com/negative-results/. Acesso em: 6 ago. 2019.

KNIGHT, Jonathan. Negative results: Null and void. Nature, [S.I.], n. 422, 2003. Disponível em:

https://www.researchgate.net/publication/10812294_Negative_results_Null_and _void. Acesso em: 7 dez. 2019.

KUHN, Thomas. The structure of scientific Revolution. 2. ed. Chicago: The University of Chicago, 1970. Disponível em: https://folk.ntnu.no/krill/biokoreferences/Kuhn\%201962.pdf. Acesso em: 6 dez. 2019.

LATOUR, Bruno. "O objetivo da ciência não é produzir verdades indiscutíveis, mas discutíveis". [Entrevista concedida ao] Jornal Correio do Povo, Porto Alegre, 11 mar. 2017. Disponível em:

https://www.correiodopovo.com.br/blogs/di\%C3\%A1logos/bruno-latour-o- 
objetivo-da-ci\%C3\%AAncia-n\%C3\%A3o-\%C3\%A9-produzir-verdadeindiscut\%C3\%ADveis-mas-discut\%C3\%ADveis-1.306155. Acesso em: 6 dez. 2019.

MATOSIN, Natalie; FRANK, Elisabeth; ENGEL, Martin; LUM, Jeremy S.; NEWELL. Negativity towards negative results: a discussion of the disconnect between scientific worth and scientific culture. Disease Models \& Mechanisms, [S.I.], v. 7, p. 171-173, 2014. Disponível em: https://dmm.biologists.org/content/dmm/7/2/171.full.pdf. Acesso em: 6 ago. 2019.

MOSLEY, Michael; LYNCH, John. Uma história da ciência. [S.I.]: Zahar, 2011. MUDRAK, Ben. Negative results: the dark matter of research. 2019. https://www.aje.com/arc/negative-results-dark-matter-research/. Acesso em: 6 ago. 2019.

ORANSKY, Ivan; MARCUS, Adam. Keep negativity out of politics. We need more of it in journals. STAT News, [S.I.], Oct. 14, 2016. Disponível em: https://www.statnews.com/2016/10/14/journals-negative-findings/. Acesso em: 6 ago. 2019.

PFEFFER, Christian; OLSEN, Bjorn R. Editorial. Journal of Negative Results in Biomedicine, [S.I.], v. 1, 2002. Disponível em:

https://jnrbm.biomedcentral.com/track/pdf/10.1186/1477-5751-1-2. Acesso em: 6 abr. 2020.

POPPER, Karl. Conjectures and refutations: The growth of scientific knowledge. New York, London: Basic Books Publishers, 1962. Disponível em: http://www.rosenfels.org/Popper.pdf. Acesso em: 10 dez. 2019.

RECHERCHE ANIMALE. With Negative Results: negative is positive! 2018. Disponível em: https://www.recherche-animale.org/en/negative-resultsnegative-positive. Acesso em 27 dez. 2019.

REWARDING negative results keeps science on track. Nature, [S.I.], v. 551, n. 7681, p. 414, 2017. DOI: 10.1038/d41586-017-07325-2.

SAYÃO, Luis Fernando, SALES, Luana Farias. O fim da teoria: o embate entre a ciência orientada por dados e a ciência orientada por hipoteses. LIINC em Revista, Rio de Janeiro, v. 15, n. 1, 2019. Disponível em: http://revista.ibict.br/liinc/article/view/4688/4135. Acesso em: 6 abr. 2019.

TIMBIE, Kelsie. The Stigma of Negative Data ... and How Preprint Servers Can Help. 2019. Disponível em: https://www.fusfoundation.org/thefoundation/news-media/blog/the-stigma-of-negative-data-and-how-preprintservers-can-help. Acesso em: 20 jan. 2020.

VAN HILTEN, Lucy Goodchild. Why it's time to publish research "failures". Elservier, [S.I.], 2015. Disponível em: 


\title{
THE INVISIBLE SCIENCE: WHY RESEARCHERS DO NOT PUBLISH THEIR NEGATIVE RESULTS?
}

\begin{abstract}
Introduction: The results of scientific research considered negative - unconfirmed hypotheses, unexpected data, inconclusive experiments and more - are always present in the flows of scientific knowledge construction, constituting an essential component for the more comprehensive and integral vision of the multiple faces of the laboratory routine. Furthermore, it is these studies that refute current ideas and consolidated hypotheses that advance science. However, negative studies do not find a high-impact formal publication medium and are not fully valued in the scientific environment and by its institutions. There are many barriers to not publishing and sharing these results. Objective: Trying to understand this phenomenon, the present study, through the observation of the scarce literature in the area, analyzes the main causes of the invisibility of negative studies. Methodology: Based on exploratory and qualitative research, the search and analysis of the scarce literature on the subject is adopted as a methodological resource, investigating and systematizing the main causes of the invisibility of negative studies. Results: As a result, the following barriers are identified and systematized: contextual, cognitive, professional, organizational and editorial. Conclusion: It is concluded that although there is a movement around the creation of journals and other platforms dedicated to negative results, these information assets still need to be valued and integrated to the scientific communication flows, providing a greater visibility to non-confirmatory studies.
\end{abstract}

Descriptors: Negative result. Scientific journal. Scientific communication. Invisible science.

\section{LA CIENCIA INVISIBLE: ¿POR QUÉ LOS INVESTIGADORES NO PUBLICAN SUS RESULTADOS NEGATIVOS?}

\begin{abstract}
RESUMEN
Introducción: los resultados de las investigaciones científicas considerados negativos - hipótesis no confirmadas, datos inesperados, experimentos no concluyentes y más siempre están presentes en los flujos de construcción del conocimiento científico, constituyendo un componente esencial para una visión más completa e integral de las múltiples caras de las rutinas de laboratorios. Además, son estos estudios los que refutan las ideas actuales y las hipótesis consolidadas que hacen avanzar la ciencia. Sin embargo, los estudios negativos no encuentran un medio de publicación formal de alto impacto y no son completamente valorados en el entorno científico y por sus instituciones. Hay muchas barreras para no publicar y compartir estos resultados. Objetivo: Al tratar de comprender este fenómeno, el presente estudio, a través de la observación de la escasa literatura en el área, analiza las principales causas de la
\end{abstract}


invisibilidad de los estudios negativos. Metodología: A partir de una investigación exploratoria y cualitativa, se adopta como recurso metodológico, la búsqueda y análisis de la escasa literatura sobre el tema, investigando y sistematizando las principales causas de la invisibilidad de los estudios negativos. Resultado: Como resultado, estas causas se categorizan y comentan en cinco categorías: barreras contextuales, cognitivas, profesionales, organizacionales y editoriales. Conclusión: Se concluye que, aunque existe un movimiento en torno a la creación de revistas y otras plataformas dedicadas a resultados negativos, estos activos de información aún deben ser valorados e integrados con los flujos de comunicación científica, lo que proporciona una mayor visibilidad a los estudios no confirmatorios.

Descriptores: Resultado negativo. Revista científica. Comunicación científica. Ciencia invisible.

Recebido em: 29.04.2020

Aceito em: 19.11 .2020 